\title{
Magnetic Hysteresis and Barkausen Noise in Plastically Deformed Steel Sheets
}

\author{
Fausto Fiorillo * (D), Michaela Küpferling and Carlo Appino \\ Istituto Nazionale di Ricerca Metrologica-INRIM, Nanoscience and Materials Dept., 10135 Torino, Italy; \\ m.kuepferling@inrim.it (M.K.); c.appino@inrim.it (C.A.) \\ * Correspondence: f.fiorillo@inrim.it; Tel.: +39-011-391-9836
}

Received: 7 December 2017; Accepted: 28 December 2017; Published: 30 December 2017

\begin{abstract}
The magnetic properties of steels are affected by plastic deformation, because the domain wall processes magnetoelastically interact with the dislocations and the residual stresses. The evolution of the magnetic hysteresis loop and its parameters with the type and degree of straining can thus provide a macroscopic signature of the underlying the mechanical and structural properties. Additional information can be achieved at a microscopic level through analysis of the Barkhausen noise, the signal generated by the stochastic flux variations associated with the discontinuous motion of the domain walls. Nondestructive methods for the structural evaluation of magnetic steels, devoted, in particular, to the investigation of work-hardening and state of internal stress following plastic straining, have therefore been developed in the literature, either through magnetic hysteresis or Barkhausen noise measurements. In this paper, we summarize significant results regarding the relationship between magnetic properties and plastic deformation in steel samples and the related experimental methods. Attention will be devoted, in particular, to the measurement and analysis of the Barkhausen noise spectral density and the way it relates to the macroscopic magnetic behavior and the structural properties.
\end{abstract}

Keywords: soft magnetic steels; coercive field; Barkhausen noise; plastic strain

\section{Introduction}

Iron and iron-based alloys spontaneously expand when ferromagnetism sets in below the Curie temperature. This effect, called "volume magnetostriction", originates in the exchange interaction and the related energy term, which is minimized at the optimal interatomic distance. The volume expansion of the cubic iron cell, of the order of some $10^{-3}$, is slightly anisotropic, showing a linear strain, around some $10^{-5}$, along the direction of the magnetization $M$. The cell elongates along such a direction, until the sum of the decreasing magnetic energy and the correspondingly increasing elastic energy attains a minimum. Conversely, if the mechanical deformation is imposed, by residual or applied stresses, the magnetoelastic interaction will influence the local direction of $\boldsymbol{M}$ and, globally, the magnetic state of the material. The magnetoelastic effect in a polycrystalline steel sample is conveniently expressed through the isotropic magnetostriction constant $\lambda_{\mathrm{s}}$, the relative elongation of the magnetically saturated sample with respect to a demagnetized state endowed with isotropically distributed domains. The energy of interaction between uniaxial stress $\sigma$ and magnetization $M$ forming an angle $\theta$ is then obtained, per unit volume, as $E_{\sigma}=(3 / 2) \lambda_{s} \sigma \sin ^{2} \theta$. It is precisely this energy term that enters the magnetic phenomenology of the plastically deformed steels. Investigating such a phenomenology has obvious direct interest for steel sheets applied as magnetic cores of electrical devices, like transformers and motors, but it has also great appeal for the nondestructive assessment of the mechanical properties of the structural steels. It is remarkable that non-oriented and grain-oriented steel sheets can achieve their present-day excellent properties only through severe cold-rolling as 
intermediate preparatory steps $[1,2]$, but their loss and permeability figures eventually suffer from the plastic strain engendered by sheet cutting and core forming [3,4]. On the other hand, both ferritic and austenitic steels undergoing structural changes upon machining and cold-working can nondestructively be tested by magnetic methods [5-9].

Besides the hysteresis loop and its parameters, a great deal of activity has been devoted in the literature to the measurement and analysis of the Barkhausen noise (BN), the fingerprint of the discontinuous motion of the domain walls through the magnetization process. Actually, BN sensing methods have been developed, besides the study of plastic straining and work-hardened state [10], for a number of nondestructive investigations on structural properties and effects in steels, like grain size [11], radiation damage [12], carbide precipitates [13], fatigue [14], surface decarburization [15], martensitic transformation [16], and welding-induced residual stresses [17].

In the present work, we shall highlight the main features of the plastic-deformation-dependent magnetic properties of steels, summarizing significant achievements of the literature and discussing the physical mechanisms lying behind such properties. We shall distinguish, on one hand, between the role of the dislocations, locally interacting with the domain walls and their motion and, on a larger scale, the effect of the residual stresses. On the other hand, we shall consider both the macroscopic material response, in terms of hysteresis loop and coercivity, and the microscopic aspects of the magnetization reversal, through analysis of the Barkhausen noise. This work contains both unpublished results by the authors and results from the literature. The latter are duly mentioned and tuned to this presentation by fully original figures.

\section{Dislocations, Residual Stress, Hysteresis Loop and Coercivity}

\subsection{Plastic Strain and Magnetization Process}

Plastic deformation of crystalline materials occurs by the motion of existing dislocations and the generation of new ones, by which atomic planes can slide one with respect to another. In Fe and Fe-based alloys, the primary slip planes are identified with the dense-packed (110) planes. Dislocations move and dislocation sources are activated on these planes at an early stage of plastic straining (stage I). With increasing deformation, secondary slip planes are activated (stage II) and the isolated dislocations created in stage I tend to intersect and pin each other, creating tangles and making deformation increasingly difficult. The yield stress is correspondingly increased (strain-hardening). In single crystals, a sharp increase of the work-hardening rate is correspondingly observed, and a correspondence is found with the concurring increase of the coercive field, as illustrated in Figure 1 for an intermediately oriented pure Fe single crystal [18]. Stage III takes place when the applied stress is high enough to enable the cross-slip of pinned dislocations between neighbouring crystalline planes, thereby reducing the work-hardening rate. A cellular dislocation structure, with piled-up dislocation surrounding near dislocation-free areas, eventually forms.

Both $180^{\circ}$ and $90^{\circ}$ domain walls (DWs) can interact with dislocations. They do this to a different extent, because the $180^{\circ} \mathrm{DWs}$ display magnetostrictive behavior only inside the wall, while $90^{\circ} \mathrm{DWs}$ generate long-range stresses. They are therefore far less mobile than the $180^{\circ} \mathrm{DWs}$. The theoretical analysis of the interaction of the Bloch walls with the stress field generated by the dislocations predicts for a single crystal an increase of the coercive field with the work-hardening of the type $H_{\mathrm{c}} \propto\left(\tau-\tau_{0}\right)^{1 / 2}$, where $\tau$ is the resolved shear stress and $\tau_{0}$ is the critical shear stress, along stage I and $H_{\mathrm{C}} \propto\left(\tau-\tau_{0}\right)$ along stages II and III [19]. This amounts to state that the coercivity is proportionally related to the dislocation density $\rho$ as $H_{\mathrm{c}} \propto \rho^{1 / 2}$. The distinction between the different deformation stages becomes blurred in polycrystalline materials, because the different orientations of the grains impose the activation of different slip planes since the inception of plastic straining, in order to preserve the mechanical coherence between neighbouring grains. Consequently, the work-hardening rate is the highest at low strains, as shown for interstitial-free (IF) steels [20] and ultra-low-carbon steels [21] of similarly small grain size $(<\mathrm{s}>=17 \mu \mathrm{m}$ and $\langle\mathrm{s}\rangle=16 \mu \mathrm{m}$, respectively), and for a very pure 
large-grained ( $<s>=800 \mu \mathrm{m}$ ) Fe sample [22]. It is observed in fact (Figure 2a) that the work-hardening $\Delta \sigma$ follows to good extent a square root dependence on plastic strain $\Delta \sigma \propto \varepsilon_{\mathrm{p}}{ }^{1 / 2}$, so that the coercive field, according to the previously introduced linear dependence $H_{\mathrm{c}} \propto\left(\tau-\tau_{0}\right)$ occurring along stages II and III in single Fe crystals, is expected to approximate the law $H_{\mathrm{c}} \propto \varepsilon_{\mathrm{p}}{ }^{1 / 2}$. Figure 3 shows that such a law approximately holds in the tensile strained fine-grained IF and ultra-low carbon steels, where different deformation stages cannot be recognized. The $H_{\mathrm{c}}$ dependence on $\varepsilon_{\mathrm{p}}$ in the large-grained extra-pure Fe sample is instead reminiscent of the response of the single crystal, with stage II following stage I above about $\varepsilon_{\mathrm{p}}=2 \%$ [22].

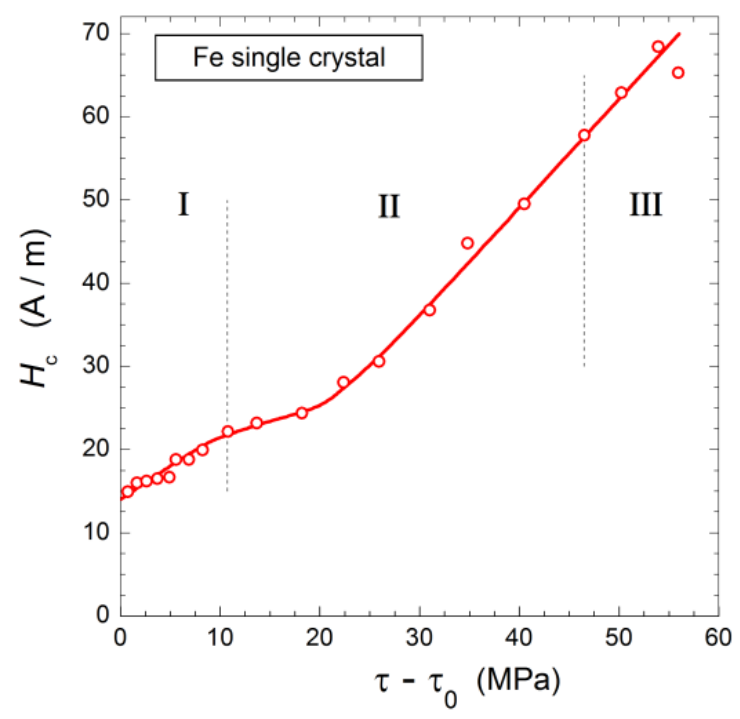

Figure 1. Coercive field versus strain-hardening of a plastically deformed intermediately oriented pure Fe single crystal. $\tau$ is the resolved shear stress and $\tau_{0}$ is the critical (yield) shear stress (adapted from [18]).

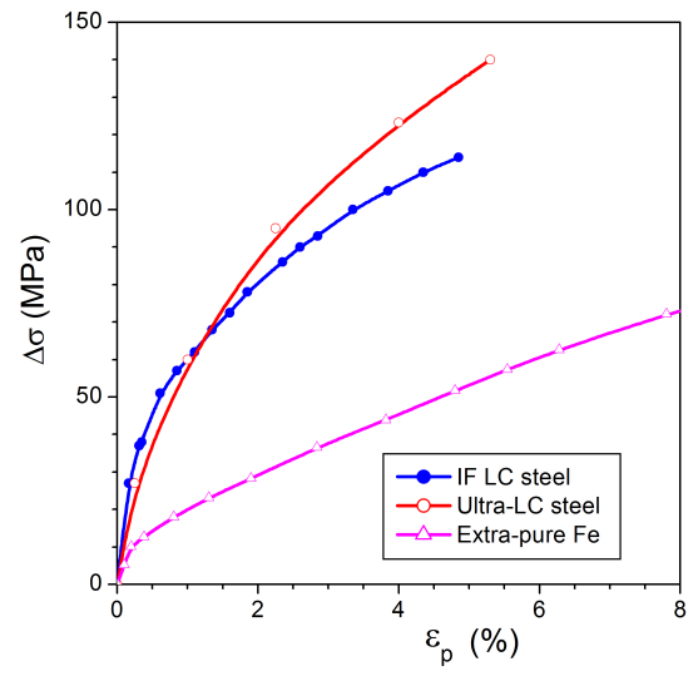

(a)

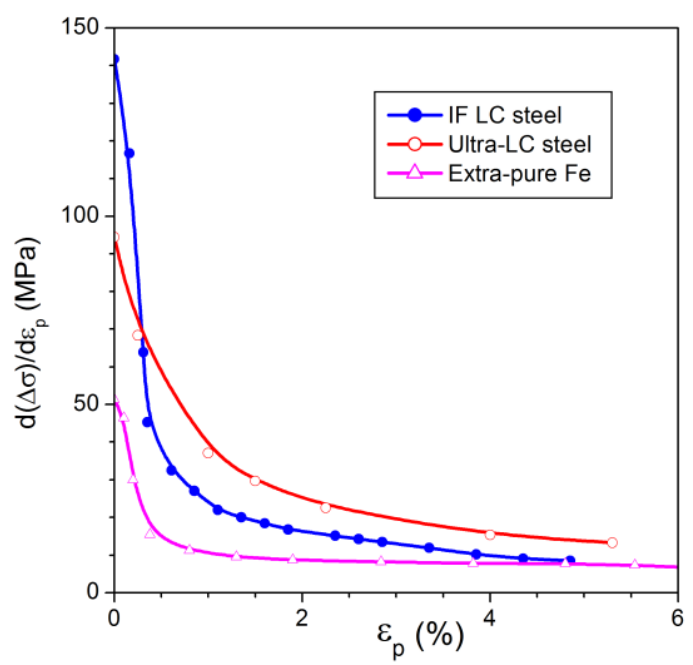

(b)

Figure 2. Work-hardening $\Delta \sigma$ (a) and work-hardening rate $\mathrm{d} \Delta \sigma / \mathrm{d} \varepsilon_{\mathrm{p}}$ (b) versus plastic strain in tensile stressed interstitial-free (IF) low-carbon steels (adapted from [20]), ultra-low-carbon steels (adapted from [21]), and extra-pure large-grained Fe samples (adapted from [22]). 


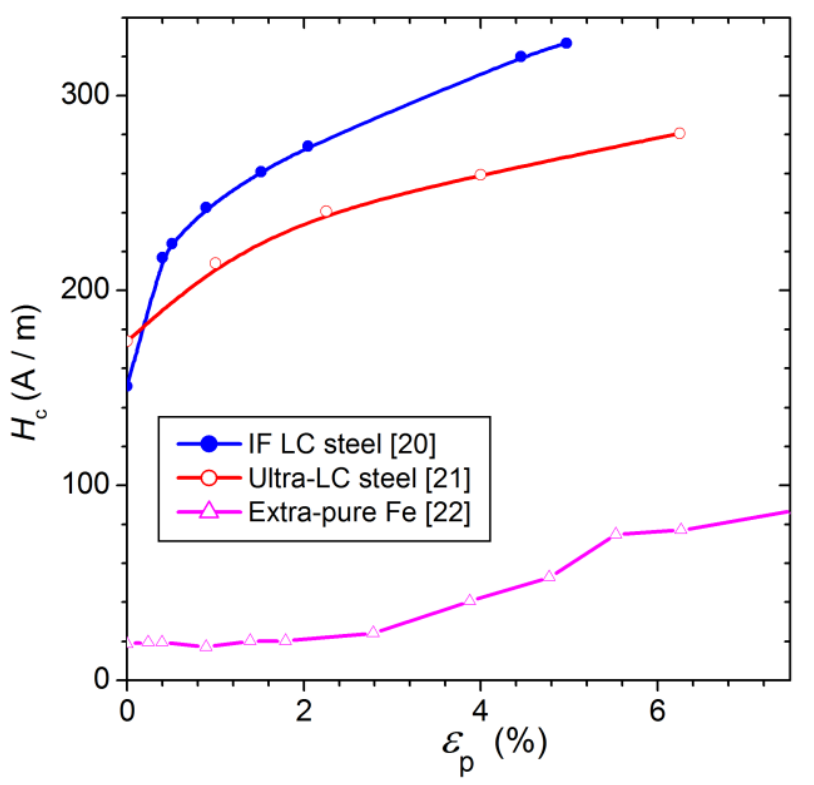

Figure 3. Work-hardening of the fine-grained IF and ultra-low carbon steels of Figure 2 brings about a dependence of the coercive field on plastic straining approximately following the law $H_{\mathrm{c}} \propto \varepsilon_{\mathrm{p}}{ }^{1 / 2}$. Deformation occurs in a rather continuous fashion and different stages cannot be discriminated. Stage I and stage II are instead recognized below and above about $\varepsilon_{\mathrm{p}} \sim 2 \%$ in the large-grained $(<s>=800 \mu \mathrm{m})$ extra-pure Fe sample. The data shown in this figure have been adapted from Refs. [20-22].

Plastic deformation of steel samples by uniform tensile stress is expectedly uniform, in the sense that residual stresses in the sample are mainly confined to the microscopic and mesoscopic scales. It has been suggested that the local residual compressive stresses are spatially prevalent against the tensile ones once the external longitudinal tensile stress is released [23]. This implies, however, that reduced magnetic hardening should occur along the transverse direction [24,25]. The resulting anisotropic magnetic behavior might be observed, for example, applying the technique discussed in [26], based on the local measurement by Hall sensors of the magnetic induction versus angle on circular samples. Residual stresses are indeed balanced, but their effects on the magnetic properties are not. The magnetization process in iron and its alloys (typically, $\mathrm{Fe}-\mathrm{Si}$ and low-carbon steels) will be slightly favoured, in general, along the direction of the stress, if this is low and tensile in nature, but it will be strongly hindered along the same direction if it is compressive. Besides the localized interaction of the domain walls with the dislocations, the plastic straining actually interferes with the domain structure through the residual mesoscopic and macroscopic stresses. A region invested by a longitudinal compressive stress will have its magnetization preferably bound to the easy axes closest to the transverse direction, with obvious detrimental effects on the motion of the domain walls when a longitudinal field is applied. This condition is well illustrated by the experimentally found evolution of the magnetic hysteresis loops of non-oriented Fe-Si sheets under tensile and compressive stresses in the elastic regime, examples of which are shown in Figure 4 [27,28]. Large detrimental effect on the soft magnetic behavior is observed in the compressive regime, where the loops exhibit, in particular, a somewhat bulged shape around the coercive field. This is due to the extra-field required for switching the magnetization, via $90^{\circ} \mathrm{DW}$ displacements, between easy axes, from the ones favored by the compressive stress to those closer to the applied field direction. The $90^{\circ} \mathrm{DWs}$, with their long-range interaction with the stress field are far less mobile than the $180^{\circ} \mathrm{DWs}$, and it is therefore not surprising a certain similitude between the hysteresis loops of the plastically deformed and the compressively stressed polycrystalline steel sheets. We show in Figure 5 the evolution of the quasi-static hysteresis loops, all measured at the same peak polarization value $J_{\mathrm{p}}=1.72 \mathrm{~T}$, up to plastic elongation $\varepsilon_{\mathrm{p}}=4.5 \%$, in the tensile strained IF steel strip samples (length $300 \mathrm{~mm}$, width $30 \mathrm{~mm}$, thickness 
$1.25 \mathrm{~mm}$ ). These strips were strained at a constant deformation rate $\mathrm{d} \varepsilon / \mathrm{d} t=1.7 \times 10^{-4} \mathrm{~s}^{-1} \mathrm{using}$ an INSTRON machine. The dependence of the loop areas (hysteresis energy loss) on $\varepsilon_{\mathrm{p}}$ is consistent with that of the coercive field reported in Figure 3. The dramatic drop of the remanent polarization value, already occurring with deformation values as low as $\varepsilon_{\mathrm{p}}=0.5 \%$, is driven by the compressive residual stress, easily engendering, in the demagnetized state, the transition of the magnetization inside the domains towards the easy axes far-off the stress (i.e., field) direction. It is remarked in Figure $5 b$, in agreement with previous findings of the literature [24,25], that all the plastically strained loops tend to cross at the same point in the second and fourth quadrants. It has been suggested that the additional field $H_{1}$ required to achieve the same polarization value attained in the unstrained material can be expressed as the product $H_{1}(\sigma, J)=h(\sigma) g(J)$ [24]. The coincidence points are assumed to be the ones where $g(J)=0$, bringing the additional field to zero. While the physical mechanism by which the condition $g(J)=0$ should arise is not clear, we observe in Figure $5 \mathrm{~b}$ that the slope of the hysteresis loop in the second quadrant, across the region surrounding the coercive field $H_{\mathrm{c}}\left(\varepsilon_{\mathrm{p}}\right)$, behaves in approximately inverse fashion with respect to the quantity $\Delta H_{\mathrm{c}}$. This is identified with good approximation with the additional contribution to the coercivity deriving from plastic straining, the field value at the intersection point being practically coincident with the coercive field of the unstrained sample. By denoting with $\mu_{\mathrm{a}}$ the average differential permeability through this region, we thus have

$$
\Delta H_{\mathrm{c}}\left(\varepsilon_{\mathrm{p}}\right) \mu_{\mathrm{a}} \sim \text { const. }
$$

The lack of internal degrees of freedom of the domain orientations brought about by the local residual stresses is the source of internal demagnetizing fields, and one may reasonably assume these to be the chief source of the remarkable decrease of $\mu_{\mathrm{a}}$ ensuing from plastic straining (e.g., from $\sim 2 \times 10^{4}$ to $\sim 7 \times 10^{3}$ ) across the $H_{\mathrm{c}}$ region on passing from $\varepsilon_{\mathrm{p}}=0$ to $\varepsilon_{\mathrm{p}}=0.5 \%$ ). If we then recognize a proportional relationship between $\Delta H_{\mathrm{c}}\left(\varepsilon_{\mathrm{p}}\right)$ and an equivalent internal demagnetizing factor $N_{\mathrm{d}} \sim 1 / \mu_{\mathrm{a}}$, we arrive at justifying Equation (1).

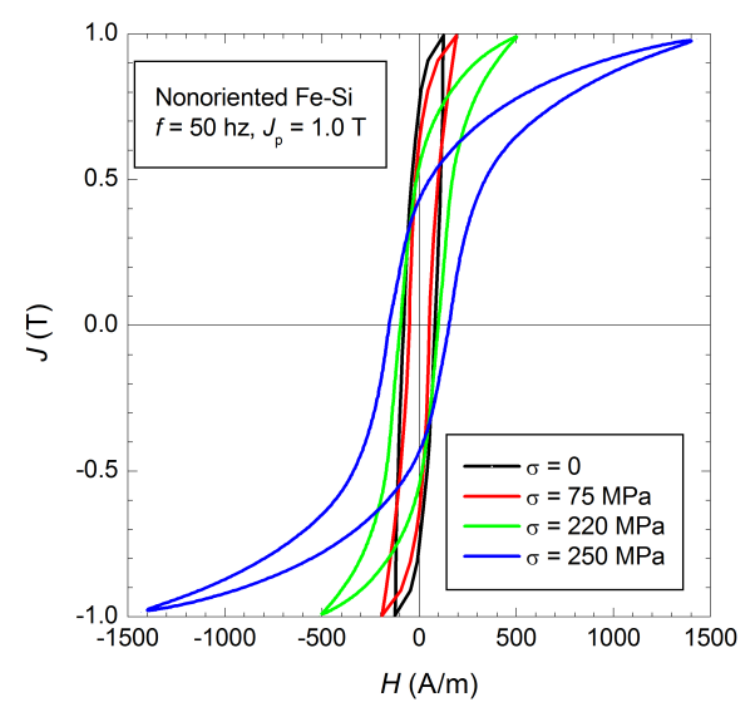

(a)

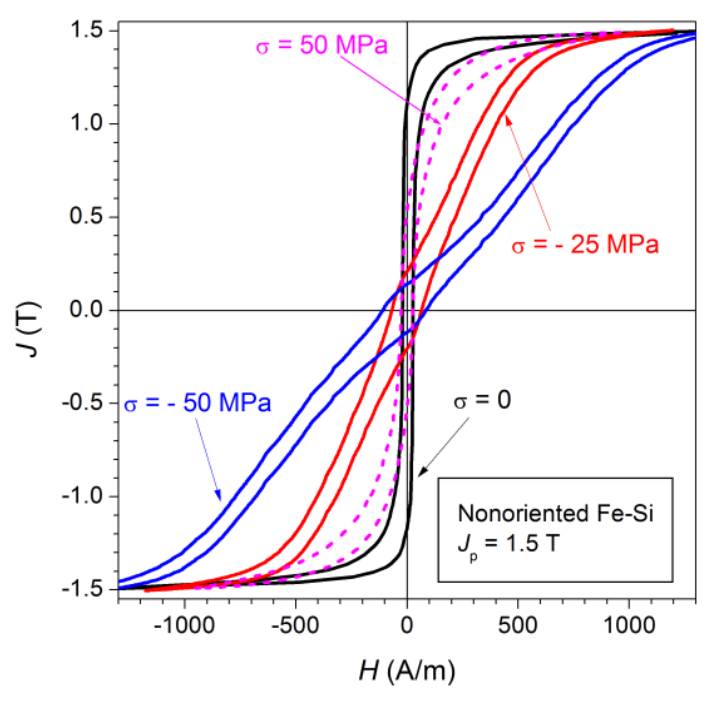

(b)

Figure 4. (a) Hysteresis loops in non-oriented Fe-Si strip samples subjected to tensile stress. The coercive field passes through a shallow minimum around $\sigma=75 \mathrm{MPa}$ (adapted from [27]). (b) Effect of compressive and tensile stresses in the range $-50 \mathrm{MPa} \leq \sigma \leq 50 \mathrm{MPa}$ on the quasi-static major hysteresis loop of a non-oriented Fe-(3 wt \%)Si sheet. A monotonic increase of the coercive field, going hand-in-hand with a decrease of permeability, is observed under increasing (conventionally negative) compressive stress (partly adapted from [28]). 


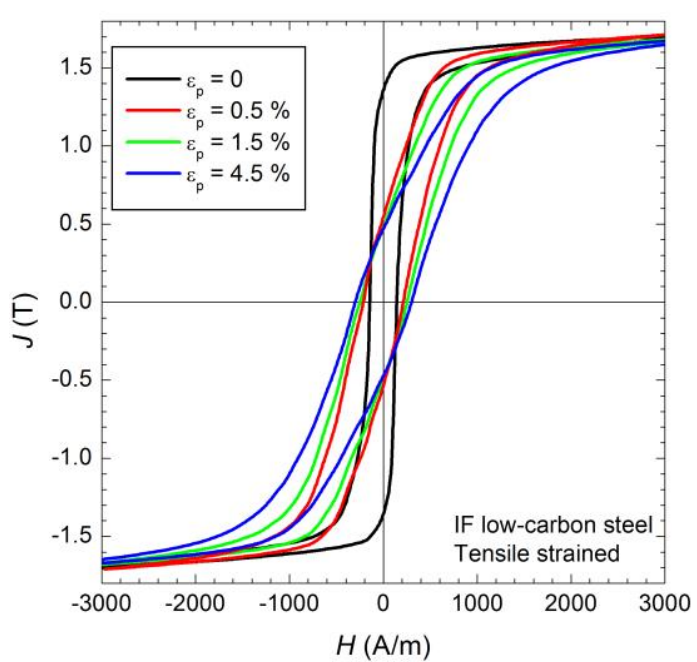

(a)

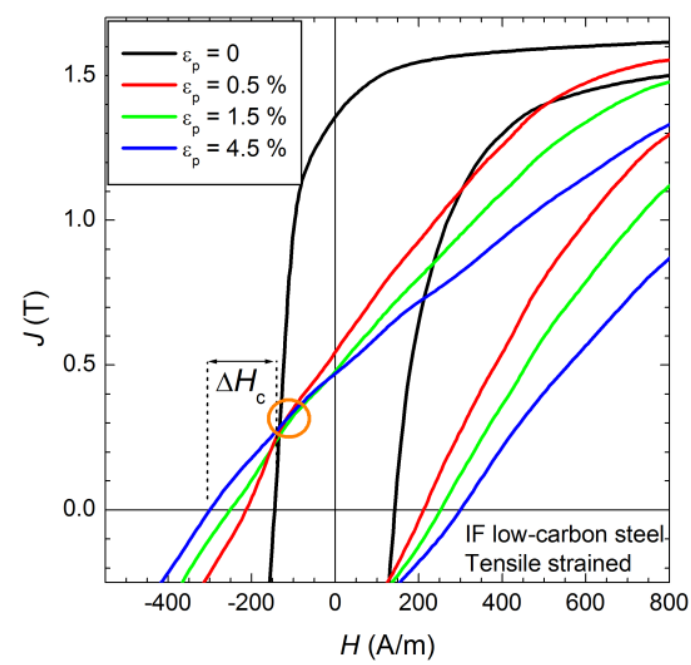

(b)

Figure 5. (a) Quasi-static major hysteresis loops measured at peak polarization $J_{\mathrm{p}}=1.72 \mathrm{~T}$ in interstitial-free (IF) steel sheets up to $\varepsilon_{\mathrm{p}}=4.5 \%$. (b) Enlarged view of the coercive field region, showing that the loops of the plastically deformed sheets tend all to cross at the same point in the second and fourth quadrants.

\subsection{Tensile Straining versus Cold-Rolling}

It is interesting to note that reduced magnetic hardening is displayed by steels strips subjected to cold-rolling, if compared with identical samples tensile strained to the same permanent elongation. The results shown in Figure 6 refer to the previously introduced IF low-carbon steel strips, subjected to a many-pass flat rolling process. It is a non-uniform deformation process, expected to generate a macroscopic residual stress profile across the strip thickness, which, under the present rolling conditions, is predicted to be of longitudinal compressive character at the surface, evolving into tensile through the strip depth [29]. If that is the case, the core of the strip is expectedly under tensile stress and magnetically softer than the surface layer, as well as of the strips strained to the same degree in tensile mode. In the latter, the local residual compressive stresses at a mesoscopic (e.g., grain size) scale, with their unfavourably oriented domains, are in fact effective in restraining the magnetization process, because they act as efficient obstacles against the propagation of the magnetization reversal. A state of macroscopic residual tension of the core of the strip along the longitudinal direction makes instead the easy axes closer to such a direction preferably occupied, thereby weakening the hindering action of the local compressive stresses. It is noted in Figure $6 \mathrm{~b}$ that the phenomenon of hysteresis loop crossing at the same $(H, J)$ coordinate in the second and fourth quadrant, previously shown for the tensile strained sheets, equally occurs in the cold-rolled sheets.

\subsection{Strain Hardening by Cutting}

Laminations in magnetic cores of electrical machines, transformers, and components are shaped by cutting and their soft magnetic properties suffer from the correspondingly introduced work-hardening. The degradation of the magnetic properties, propagating up to a few millimeters from the cutting line towards the sheet interior, can be large, as found, for example, in the teeth of the stator core of the rotating machines [30]. The better the material quality, the larger the detrimental effect of cutting. In order to practically cope with this problem, the conventional guillotine punching is often substituted by the time consuming water cutting or spark erosion methods. We see in Figure 7a how punching deteriorates the soft magnetic response of a $0.638 \mathrm{~mm}$ thick non-oriented Fe-Si strip when passing from the standard Epstein width $w=30 \mathrm{~mm}$ to $w=5 \mathrm{~mm}$. The coercive field and the quasi-static energy loss at $J_{\mathrm{p}}=1.5 \mathrm{~T}$ are found to increase from $60 \mathrm{~A} / \mathrm{m}$ to $97 \mathrm{~A} / \mathrm{m}$ and from $51.9 \mathrm{~mJ} / \mathrm{kg}$ to $62.9 \mathrm{~mJ} / \mathrm{kg}$, 
respectively. The somewhat inflated behavior of the loop around the coercivity region is reminding of the behavior of the loops after plastic straining shown in Figures 5 and 6.

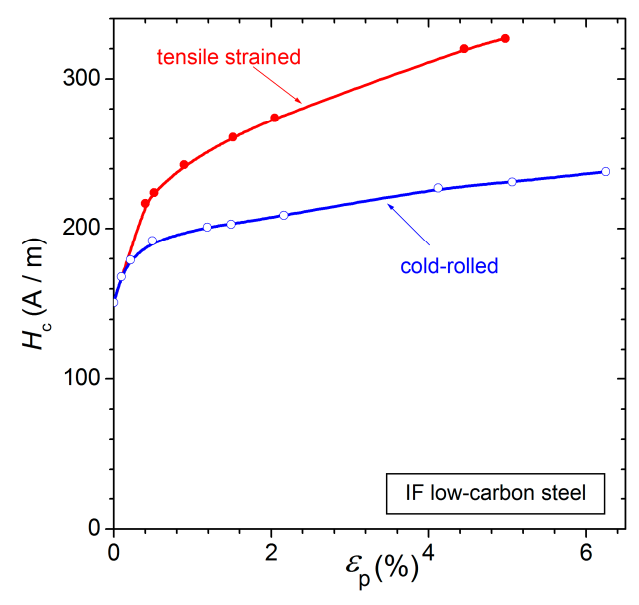

(a)

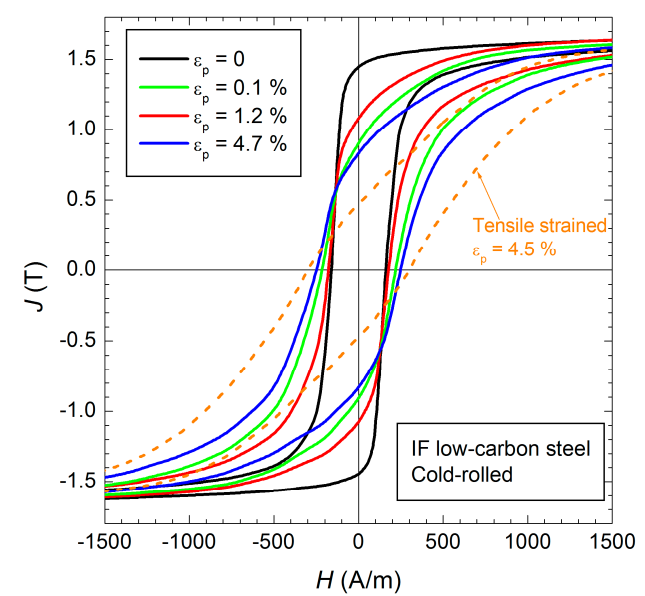

(b)

Figure 6. Magnetic hardening in cold-rolled IF low-carbon steel strips. (a) The coercive field shows, for a same plastic elongation, milder increase upon cold-rolling than with tensile straining (adapted from [20]). (b) The quasi-static major hysteresis loops $\left(J_{p}=1.72 \mathrm{~T}\right)$ accordingly show slower decrease of permeability and remanence with respect to the strips tensile strained to the same elongation level.

Modeling the detrimental effect of cutting on magnetic losses is typically pursued through phenomenological approaches and numerical methods, where the properties of the work-hardened narrow band running along the damaged sheet edges compound with the contribution of the undamaged inner region of the sheet [3,30-32]. By approximating the fall of the magnetization level across the work-hardened bands at the edges of the strip with a step-like function, we assign, for any given field $H$, the magnetization values $J_{\mathrm{pc}}$ to the hardened side bands of width $L_{\mathrm{c}}$ and the value $J_{\mathrm{p} 0}>J_{\mathrm{pc}}$ to the inner region of width $w_{0}=w-2 L_{\mathrm{c}}$ (see inset of Figure $7 \mathrm{~b}$ ). The measured magnetization $J_{\mathrm{p}}$ under defined $H$ value is given by the weighted sum of $J_{\mathrm{p} 0}$ and $J_{\mathrm{pc}}$, according to

$$
J_{\mathrm{p}}=J_{\mathrm{p} 0}-\left(J_{\mathrm{p} 0}-J_{\mathrm{pc}}\right) \cdot \frac{2 L_{\mathrm{c}}}{w}
$$

for $w \geq 2 L_{\mathrm{c}}$, and by $J_{\mathrm{p}}=J_{\mathrm{pc}}$ for $w \leq 2 L_{\mathrm{c}}$. By measuring the magnetization curve at two strip widths, one can derive the quantities $J_{\mathrm{p} 0}$ and $\left(J_{\mathrm{p} 0}-J_{\mathrm{pc}}\right) L_{\mathrm{c}}$ as a function of $H$. We then obtain a family of curves for a convenient number of $H$ values, which describe the dependence of $J_{\mathrm{p}}$ on $w$, until the minimum width $2 L_{\mathrm{c}}$. The overall behavior of these curves permits one to estimate $L_{\mathrm{c}}$, which is typically of the order of 1-3 mm, depending on the cutting method [3,33]. Using then the same simplifying scheme leading to Equation (2), we write for the hysteresis loss $W_{\mathrm{h}}$ at given $J_{\mathrm{p}}$ value

$$
W_{\mathrm{h}}=W_{\mathrm{h} 0}+\left(W_{\mathrm{hc}}-W_{\mathrm{h} 0}\right) \cdot \frac{2 L_{\mathrm{c}}}{w}
$$

where $W_{\mathrm{h} 0}$ and $W_{\mathrm{hc}}$ are the energy loss densities in the unscathed strip portion of width $w_{0}$ and the work-hardened edge bands, respectively, $L_{\mathrm{c}}$ has been estimated via Equation (2), and $w \geq 2 L_{\mathrm{c}}$. The overall dependence of the normalized energy loss $W_{\mathrm{h} \text {,norm }}=W_{\mathrm{h}} / W_{\mathrm{h} 0}$ on $w$ predicted by Equation (3) is shown for $J_{\mathrm{p}}=1.5 \mathrm{~T}$ in Figure $7 \mathrm{~b}$ (solid lines). The curves refer to both punched and water-jet cut M440-65A (thickness $d=0.638 \mathrm{~mm})$ and M440-50A $(d=0.470 \mathrm{~mm})$ Fe-Si strips and agree with the evolution of the experimental $W_{\mathrm{h} \text {,norm }}$ values (symbols). The lower magnetic deterioration brought about by water-jet cutting with respect to punching is apparent. 


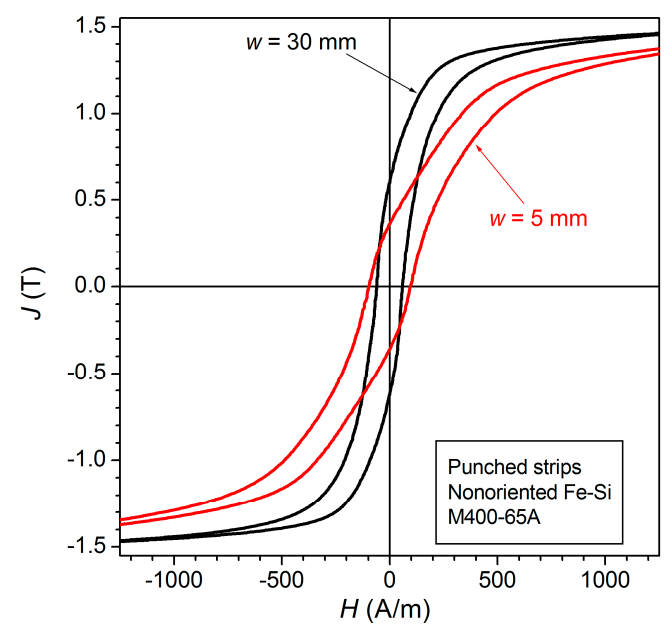

(a)

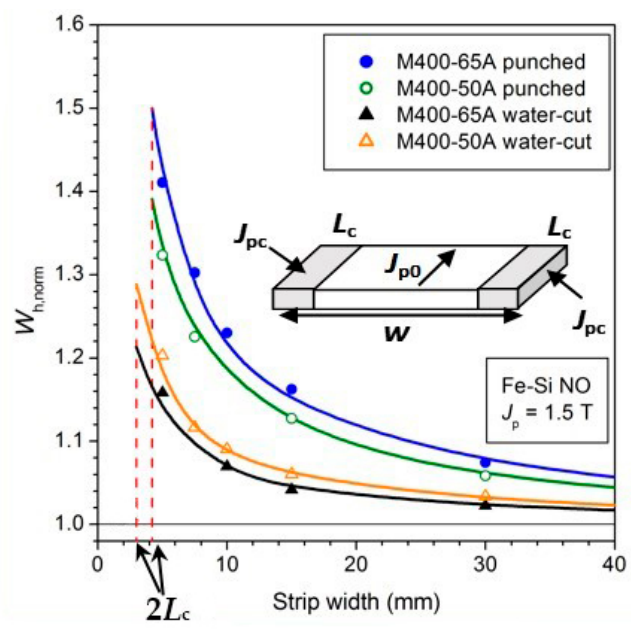

(b)

Figure 7. (a) Effect of sheet cutting on the quasi-static hysteresis loop of non-oriented Fe-Si alloys $\left(J_{\mathrm{p}}=1.5 \mathrm{~T}\right)$. The loops in this figure have been measured on guillotine-cut strips of thickness $d=0.638 \mathrm{~mm}$ and widths $w=30 \mathrm{~mm}$ and $w=5 \mathrm{~mm}$. (b) Increase of hysteresis energy loss $W_{\mathrm{h}}$ versus decrease of strip width $w$ in two different types of non-oriented Fe-Si sheets $(d=0.470 \mathrm{~mm}$ and $d=0.638 \mathrm{~mm})$, subjected either to punching or water cutting. The loss figure is normalized to the value expected for a very large sheet, whose properties are negligibly affected by cutting. Symbols: experimental $W_{\mathrm{h}}$ values. Continuous lines: prediction by Equation (3). The theoretical curves attain an upper limiting value at the strip widths where the material is assumed to be fully degraded by cutting (adapted from [33]). The inset shows the assumed schematic subdivision of the strip width between damaged (width $2 L_{\mathrm{c}}$ ) and pristine regions.

\section{Barkhausen Noise in Plastically Deformed Steels}

\subsection{Properties and Measurement of the Barkhausen Noise}

The previously discussed dependence of the macroscopic magnetic properties on type and magnitude of plastic deformation has an obvious counterpart in the evolution of the magnetization process at a microscopic/mesoscopic level, where pinning and unpinning of the DWs and rearrangements of the domain structures are affected by the magnetoelastic interactions with the dislocations and the residual stress pattern. These mechanisms have an intrinsic stochastic character and the analysis of the induction derivative, as detected by a linked coil or a suitable sensor, reveals a random contribution superposed to a continuous signal. By choosing a suitably low magnetizing frequency and appropriate high-pass filtering, the random signal (Barkhausen noise, BN) generated by the discontinuous motion of the DWs (Barkhausen jumps) can be isolated and retrieved, thereby providing information on the magnetization process and the way it is related to the macroscopic hysteresis properties.

Detection and analysis of the BN have long since been employed for nondestructive sensing of many structural phenomena and physical effects in steels, including austenitic steels, exploiting the dependence of the significant parameters of the noise on specific macroscopic parameters of the material [10-17]. Actually, BN measurements do not provide absolute quantities and one generally looks at the variation of the $\mathrm{BN}$ properties with respect to a reference state. This limitation often compounds with ambiguities deriving from the specifically chosen measuring method. The typical measuring approach consists in applying a periodic triangular field of suitably high peak amplitude by a solenoid or a soft magnetic yoke, and in detecting the secondary signal, either by a secondary coil or an inductor endowed with a soft core (permalloy, soft ferrite) placed on the sample surface. The usual exciting frequency $f_{\mathrm{m}}$ is in the range $0.1 \mathrm{~Hz}-10 \mathrm{~Hz}$ and, in order to separate the noise from the continuous secondary signal, high-pass filtering is adopted; the higher $f_{\mathrm{m}}$ the higher the cutoff 
frequency. By filtering, however, one may deprive the noise signal of significant information. In most cases the true rms value of the Barkhausen signal versus time (i.e., applied field) is measured along the cycle, and its envelope, resulting from an appropriately large number of averages, is empirically connected through its parameters (e.g., maximum value, integral over the half-period, pulse counting, amplitude distribution, etc.) to the specific state or property of the material. The effect of plastic deformation on these parameters is apparent, though the direction of the involved changes is not universally shared and contradictory results are often reported in the literature. We see in Figure 8 , taken from [34], the case of Armco Fe sheets subjected to either 10\% cold-rolling or 5\% tensile deformation, whose $\mathrm{BN}$ envelopes versus applied field show quite different behaviors, with the tensile strained samples showing a little increase of their Barkhausen emission with respect to the unstrained samples. On the other hand, the results reported in Figure 9a, concerning ultra-low carbon steels, bring to light a drastic decrease of the BN in 10\% tensile strained samples [21].

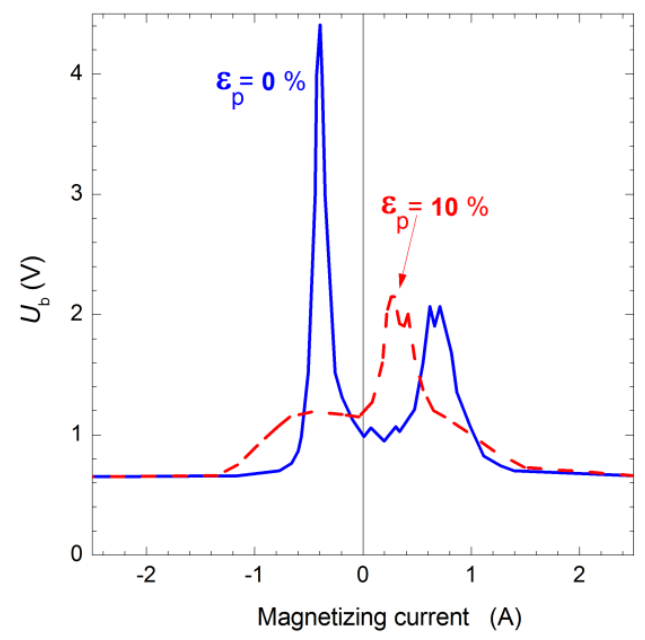

(a)

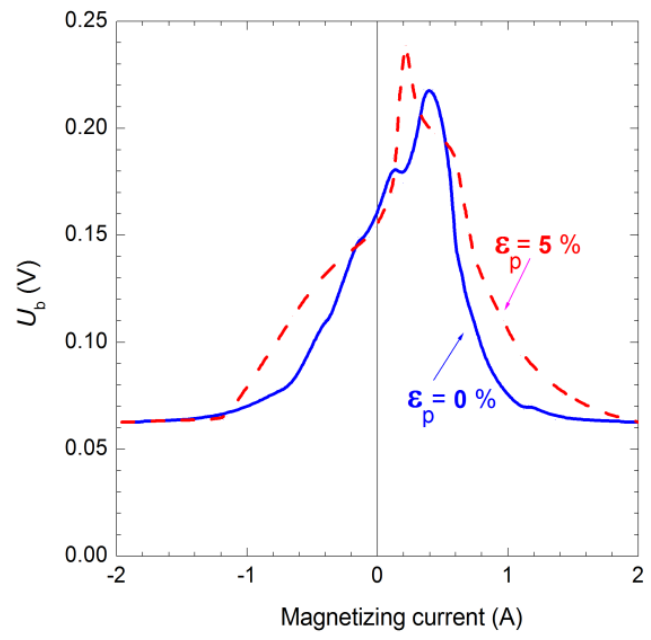

(b)

Figure 8. Envelope of the Barkhausen noise versus applied field in Armco Fe sheets before and after plastic deformation by cold-rolling (a) and tensile straining (b). Adapted from [34].

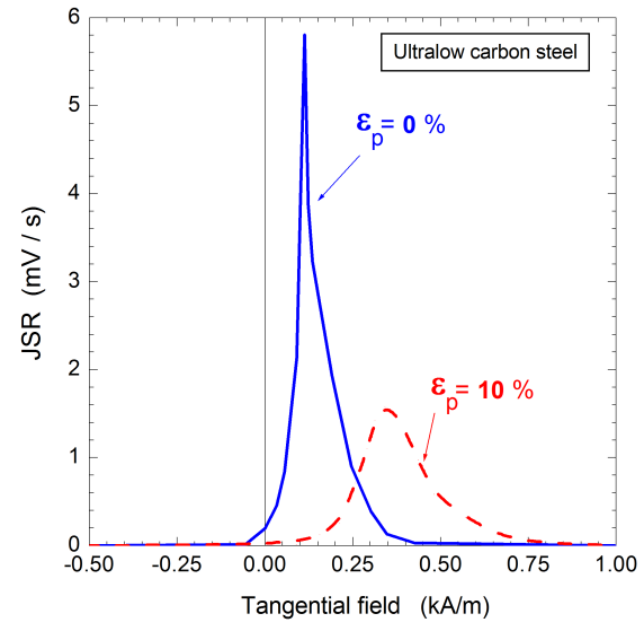

(a)

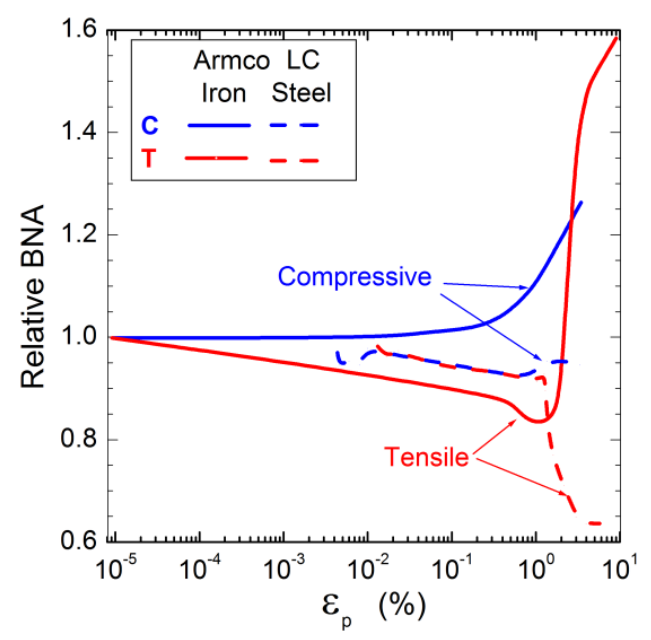

(b)

Figure 9. (a) The Barkhausen jump sum rate (JSR) in $1 \mathrm{~mm}$ thick ultra-low carbon steel sheets tensile strained up to $10 \%$ (adapted from [21]). (b) Maximum amplitude of the Barkhausen noise versus plastic deformation measured in Armco Fe and low-carbon steel bar samples. Straining is performed applying either compressive or tensile stresses (adapted from [7]). 
A further example is given in Figure 9b, regarding low-carbon steels and Armco Fe bar samples stressed both in compression and tension. Here the BN response to the deformation shows opposite trends in the two materials [7].

The objective of achieving an easily handled and rapidly analyzed Barkhausen signal for nondestructive testing can often impose relatively high magnetizing frequencies besides little or no control of the macroscopic polarization rate $\mathrm{d} J / \mathrm{d} t$. Consequently, a more or less extended low-frequency portion of the noise spectrum, masked by the continuous signal and its harmonics, is neglected. At the same time, ignoring the role of $\mathrm{d} J / \mathrm{d} t$ along the hysteresis loop can lead to ambiguities and incongruous conclusions. It is generally found, for example, that the Barkhausen emission passes through a relatively sharp maximum value around the coercive field (see Figures $8 \mathrm{~b}$ and 9a) under a triangular exciting field. The $\mathrm{BN}$ is concentrated in this region because the differential permeability and $\mathrm{d} J / \mathrm{d} t$ both attain here their highest values and the noise non-stationarity is enhanced. It was actually stated in the past that a physically meaningful approach to $\mathrm{BN}$ is better achieved by investigating the random signal as function of the magnetization level instead of the applied field level, that is, the state of the material in place of an external parameter [35]. In addition, the involved DW processes are investigated on equal footing at different induction levels only by keeping the macroscopic $\mathrm{d} J / \mathrm{d} t$ constant (i.e., triangular $J(t)$ ) [36]. Under such conditions, the resulting profile of the BN, typically identified with the noise power $P$ or its square root (true rms value) on traversing the hysteresis loop becomes dramatically different with respect to the one obtained with triangular field, as illustrated in Figure 10. This figure shows the behavior of the BN power over the half period in a non-oriented $\mathrm{Fe}-\mathrm{Si}$ sheet magnetized in the polarization interval $J_{\mathrm{p}}= \pm 1.17 \mathrm{~T}$ under both triangular $H(t)\left(H_{\mathrm{p}}= \pm 130 \mathrm{~A} / \mathrm{m}\right)$ and triangular $J(t)\left(\mathrm{d} J / \mathrm{d} t=3.5 \times 10^{-2} \mathrm{~T} / \mathrm{s}\right)$ at a frequency around $0.01 \mathrm{~Hz}$. Each point of the profile is the result of noise averaging over a sufficiently high number of successive hysteresis cycles, which realize locally the stationary conditions of a statistical ensemble. However, by imposing a triangular $H(t)$ waveform, the hysteresis loop is traversed with rapidly changing magnetization rate, peaking around the coercive field, where the differential permeability $\mu_{\text {diff }} \cong \mathrm{d} J / \mathrm{d} H$ is maximum. Consequently, the BN power is compressed inside a narrow field (time) interval and it is quite difficult to distinguish between the effect of the impressed magnetization rate and that of $\mu_{\text {diff. }}$. It is apparent in Figure 10 how with constant $\mathrm{d} J / \mathrm{d} t$ one can highlight and meaningfully compare the specific features of the magnetization process at different points of the magnetization curve. It is noted, in particular, that $P$ attains its maximum value close to the region where $d \mu_{\text {diff }} / d J$ is maximum, because of the rearrangement of the domain structure following the passage through the remanence. It was actually shown in [35] that the phenomenological relationship $P=a \mu_{\text {diff }}+b\left(\mathrm{~d} \mu_{\text {diff }} / \mathrm{d} J\right)$, with $a$ and $b$ constants, applies in grain-oriented and non-oriented sheets. Making the measurements under constant $\mathrm{d} J / \mathrm{d} t$ appears then a natural way to unambiguously assess the role of plastic straining on the noise.

\subsection{The Spectral Density of the Barkhausen Noise}

The measurement of the $\mathrm{BN}$ profile across the hysteresis loop has phenomenological meaning and can be empirically exploited for the sake of nondestructive testing. However, it offers relatively minor physical insight on the underlying magnetization process, which is better revealed by analyzing the spectral content of the noise signal. In this context, we define the spectral density of the noise $S(f)$, related to the noise power by the equation

$$
P=\int_{0}^{\infty} S(f) \mathrm{d} f .
$$




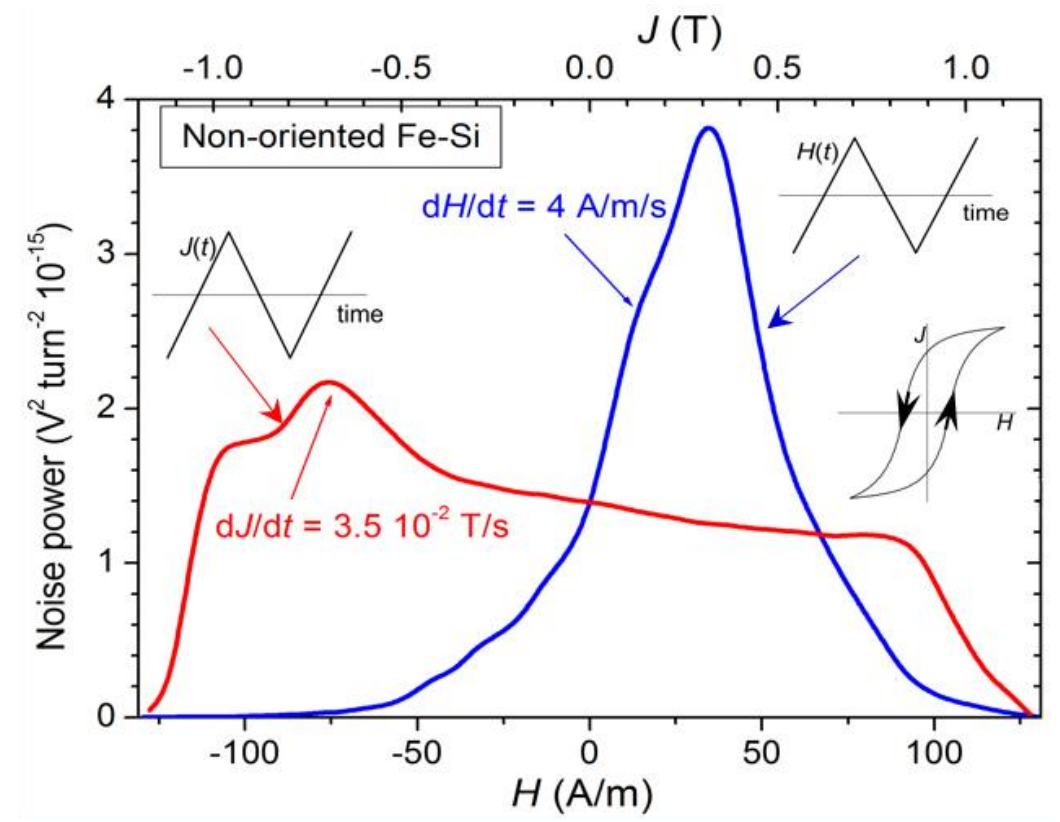

Figure 10. Profile of the Barkhausen noise power measured along a half hysteresis loop with peak polarization values $J_{\mathrm{p}}= \pm 1.17 \mathrm{~T}$ in an Fe-(3 wt \%)Si sheet under either triangular applied field waveform $H(t)$ or triangular polarization $J(t)$ at a frequency of about $0.01 \mathrm{~Hz}$. With triangular field, the Barkhausen pulses are crowded inside a relatively restricted time (field) interval around the coercive field, as imposed by the non-linear $J(H)$ behavior (partly adapted from [35]).

We show in Figure 11 the spectral densities of the BN measured in the IF low-carbon steels for different levels of plastic deformation in tensile strained and cold-rolled sheets. The spectra have been obtained under constant polarization rate $\mathrm{d} J / \mathrm{d} t=0.1 \mathrm{~T} / \mathrm{s}$ between the peak polarization values $J_{\mathrm{p}}= \pm 1.2 \mathrm{~T}$. The $300 \mathrm{~mm}$ long strip samples are placed between the pole faces of a double-C soft yoke and the field is supplied by means of an enwrapping solenoid, supplied by an appropriately shaped and low-pass filtered current waveform, such as to impress the desired constant $\mathrm{d} J / \mathrm{d} t$ value. The noise is detected either by a couple of 200-turn windings connected in series opposition located at a distance of $90 \mathrm{~mm}$ or by a C-yoke of $\mathrm{Mn}-\mathrm{Zn}$ ferrite endowed with a 100-turn pickup coil, whose pole faces are placed on the strip surface. To note that, by using the two counter-wound far-apart pickup coils, one can compensate the continuous signal without interfering with the noise signal. Details on the measuring method, including the analysis in the frequency domain, and setup are given in [37]. Figure 11 shows that plastic deformation affects in opposite fashion the low-frequency and high-frequency behavior of $S(f)$, which is depressed below the $\mathrm{kHz}$ range and increased at high frequencies, where it attains a slope close to $1 / f^{2}$.

The direct time observation of the BN reveals a structure made of stochastically distributed magnetization reversals (Barkhausen avalanches), whose statistical properties are connected with the parameters of the hysteresis loop. The experiments show that the Barkhausen reversals originate from DW instabilities occurring at pinning points and propagating along the direction of the applied field. The dynamics of the propagation process adjusts to the balance between applied and internal fields, the latter being chiefly of magnetostatic and eddy current origins. The reversals are interpreted in the statistical theory of the BN in terms of clustering by correlation of elementary Barkhausen jumps. Structural changes of the material and the related effects on the internal fields can modify the degree of clustering, that is, the amplitude and duration of the avalanches. In this respect, a fundamental role is played by the demagnetizing fields, which contrast clustering by restraining the propagation process [38]. The spectral density of the noise is consequently affected. 


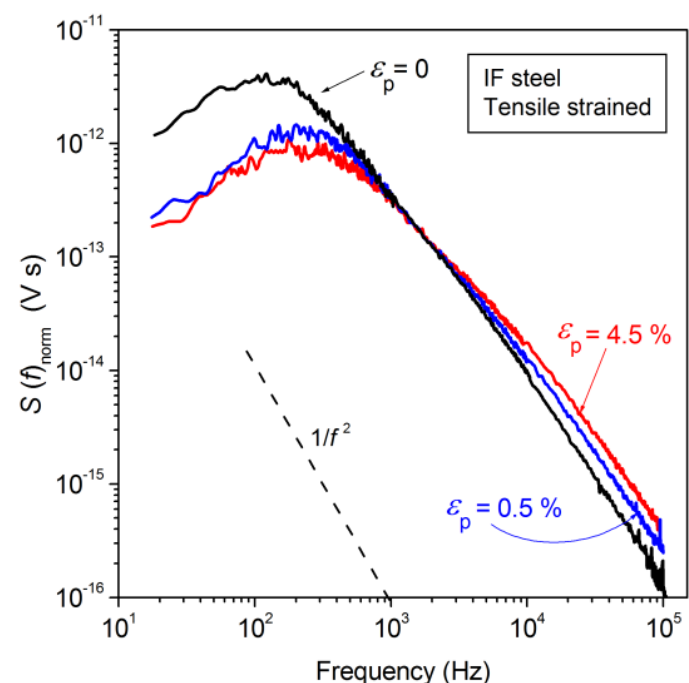

(a)

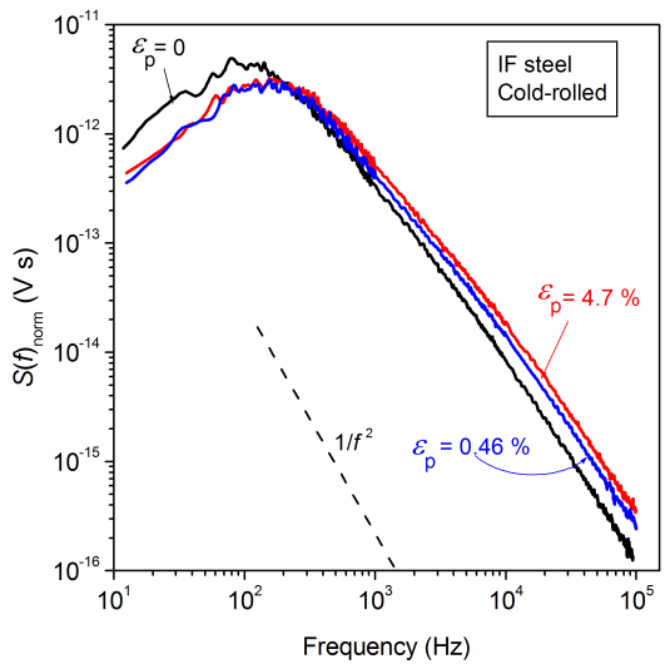

(b)

Figure 11. Spectral density of the $B N$ in IF low-carbon steel sheets magnetized at the constant polarization rate $\mathrm{d} J / \mathrm{d} t=0.1 \mathrm{~T} / \mathrm{s}$ between $J_{\mathrm{p}}= \pm 1.2 \mathrm{~T}$. The measured value of $S(f)$ is normalized with respect to the flux rate $\mathrm{d} \Phi / \mathrm{d} t$ and the number of turns squared $N^{2}$ of the pickup coils $S(f)_{\text {norm }}=S(f) /\left(\mathrm{d} \Phi / \mathrm{d} t \cdot N^{2}\right)$. Plastic strain engenders, both in tensile strained (a) and cold-rolled sheets $(\mathbf{b})$, a decrease of the low-frequency portion of the spectrum and an increase at high frequencies. Tensile strain affects to larger extent, for a same permanent elongation $\varepsilon_{\mathrm{p}}$, the low-frequency dependence of $S(f)$.

According to the theory [35], we assume that all the magnetization changes are accomplished by the discontinuous motion of the DWs and we associate with each elementary Barkhausen jump a flux variation $\Delta \Phi$. The average flux change reversed by a cluster is expressed as $\Delta \bar{\Phi}_{c}=\rho \Delta \bar{\Phi}$, where $\rho$ is the average number of elementary jumps lumped in a cluster. If $v_{\mathrm{g}}$ is the average number of clusters in unit time, generating the macroscopic flux rate $\dot{\Phi}$, and $\tau_{0}$ is the average time interval between subsequent elementary events inside the cluster, the theory predicts, as fully discussed in [35], the following expression for the spectral density of the noise

$$
S(f)=4 \dot{\Phi} \cdot \Delta \bar{\Phi}_{\mathrm{c}} \frac{1}{1+\left(v_{\mathrm{g}} / 2 \pi\right)^{2} / f^{2}} \cdot \frac{1}{1+f^{2}\left(2 \pi \rho \tau_{0}\right)^{2}}
$$

where $\rho \tau_{0}$ is the average duration of a cluster. Equation (5) describes a function endowed with low and high cutoff frequencies $f_{1}=v_{\mathrm{g}} / 2 \pi$ and $f_{2}=1 / 2 \pi \rho \tau_{0}$, respectively, and a frequency dependence $S(f) \propto f^{2}$ when $f<<f_{1}$ and $S(f) \propto 1 / f^{2}$ when $f>>f_{2}$. It is noted that the macroscopic flux rate $\dot{\Phi}$ appears as a multiplying factor in Equation (5), further showing how good a simplification is to measure the noise under constant $\mathrm{d} J / \mathrm{d} t$ value. The gross features of the BN spectra and their dependence on plastic strain, shown in Figure 11 for the case of IF low-carbon steel sheet, are predicted by Equation (5), although the experimental low-frequency portion has somewhat limited extension, as imposed by the experimental requirement of a not too low magnetizing frequency $\left(f_{\mathrm{m}} \sim 0.02 \mathrm{~Hz}\right.$ in the present case). In agreement with the experimental $S(f)$ behavior and its theoretical formulation, it is convenient to separately analyze the low and high frequency ranges, exploiting the ubiquitously imposed condition of constant $\dot{\Phi}$ value. For $f<<f_{2}$ Equation (5) can be written as

$$
S(f)=4 \dot{\Phi} \cdot \Delta \bar{\Phi}_{\mathrm{c}} \frac{1}{1+\left(v_{\mathrm{g}} / 2 \pi\right)^{2} / f^{2}} .
$$


This equation shows that if the average size $\Delta \bar{\Phi}_{\mathrm{c}}$ of the clusters is decreased, as expected upon plastic straining, implying and increase of their number in unit time $v_{\mathrm{g}}, S(f)$ is decreased at low frequencies. This is quantified in Figure 12a by the behavior of the low-frequency noise power $P=\int_{10 \mathrm{~Hz}}^{200 \mathrm{~Hz}} S(f) \mathrm{d} f$ versus $\varepsilon_{\mathrm{p}}$. Plastic deformation has a detrimental effect on the propagation of the Barkhausen reversal, because number and strength of the pinning centers are increased and the internal demagnetizing fields become stronger, breaking up the avalanches. Such an effect is stronger after tensile straining than after cold-rolling and is paralleled by a correspondingly stronger drop of permeability and remanence (see Figure $6 \mathrm{~b}$ ). It can be associated with the previously remarked different patterns of residual stresses expected to arise from these different deformation modes.

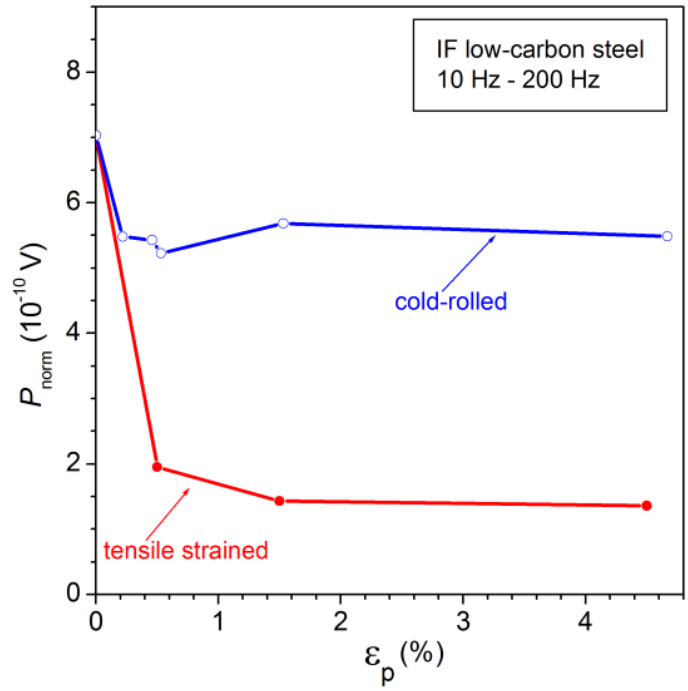

(a)

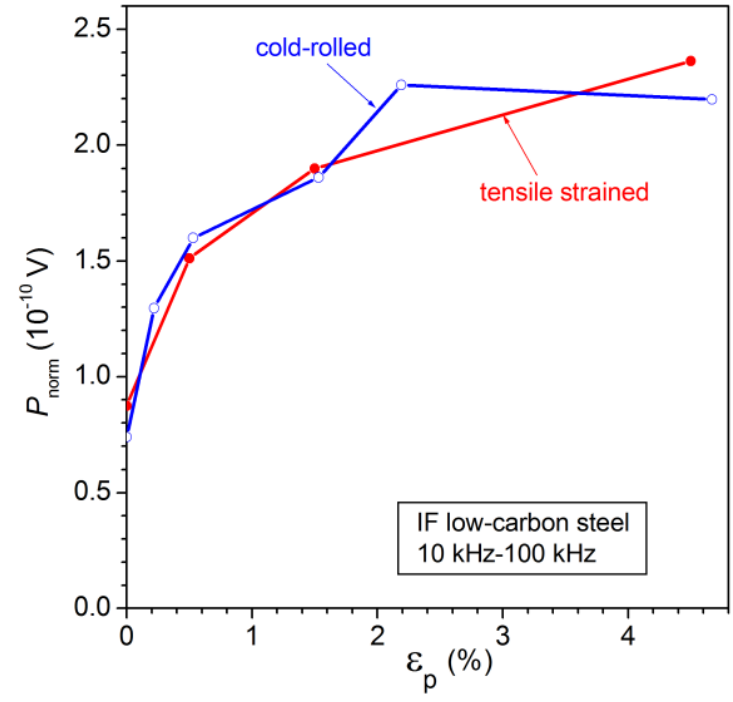

(b)

Figure 12. The noise power $P_{\text {norm }}$ shows opposite trends versus plastic deformation when obtained by integrating the normalized spectral density of the noise $S(f)_{\text {norm }}=S(f) /\left(\mathrm{d} \Phi / \mathrm{d} t \cdot N^{2}\right)$ in the low-frequency range $10 \mathrm{~Hz}-200 \mathrm{~Hz}$ (a) or in the high-frequency range $10 \mathrm{kHz}-100 \mathrm{kHz}$ (b). The low-frequency power is mainly determined by the ease of propagation of the Barkhausen avalanches, less hindered in cold-rolled sheets. The high-frequency power, quite independent of the deformation mode, is related to the fine structure of the noise, and is chiefly sensitive to the domain wall (DW) displacements occurring at and close to the sheet surface.

Actually, the noise spectrum accommodates increasing low-frequency contributions from increasingly inner layers of the sheet, very fast local flux variations being shielded to detection at the surface by the eddy currents. The cold-rolled sheets, with their residual favourable tensile stress in the core, are then the seat of detected $\Delta \bar{\Phi}_{\mathrm{c}}$ variations larger than the tensile stressed samples and have correspondingly higher $P$ value. If we consider instead the high-frequency noise power $P=\int_{10 \mathrm{kHz}}^{100 \mathrm{kHz}} S(f) \mathrm{d} f$, we obtain the behaviors shown in Figure 12b, which are quite independent of the deformation mode. According to Equation (5), the spectral density at high frequencies $\left(f>>f_{2}\right)$ can be written as

$$
S(f)=4 \dot{\Phi} \cdot \frac{1}{4 \pi^{2} \rho \tau_{0}} \cdot \frac{\Delta \bar{\Phi}}{\tau_{0}} \cdot \frac{1}{f^{2}}
$$

highlighting the $1 / f^{2}$ behavior and the dependence on the amplitude of the elementary Barkhausen jumps $\Delta \bar{\Phi}$. The high-frequency portion of the spectrum reflects the fine structure of the noise, that is, the elementary jumps and their sequence in the avalanche and the preponderant role of the events occurring at and close to the surface, where flux variations are little shielded by the eddy currents. The high-frequency noise is therefore mainly related to the local interactions between dislocations and DWs in the surface/subsurface region, independent of the specific macroscopic pattern of the 
residual stress. It is noted that the high-frequency noise power (Figure 12b), the coercive field, and the work-hardening all follow a similar approximate square root dependence on the plastic strain $\varepsilon_{\mathrm{p}}$.

The here discussed BN phenomenology is qualitatively confirmed by the results obtained substituting the pickup coils with the $\mathrm{C}$-shaped $\mathrm{Mn}-\mathrm{Zn}$ ferrite sensor, a half-ring with pole faces of area $20 \mathrm{~mm} \times 5 \mathrm{~mm}$ placed on the sheet surface. The ferrite exhibits constant initial permeability up to about $200 \mathrm{kHz}\left(\mu_{\mathrm{r}} \sim 5 \times 10^{3}\right)$ and operates as a linear transducer for the BN. In addition, by working in the reversible regime, it is not the source of additional magnetization noise. Figure 13, showing the spectral densities measured by the ferrite sensor in the starting sheet and after either cold-rolling or tensile straining up to elongations around $4.5 \%$, is in good qualitative agreement with the results reported in Figure 11.

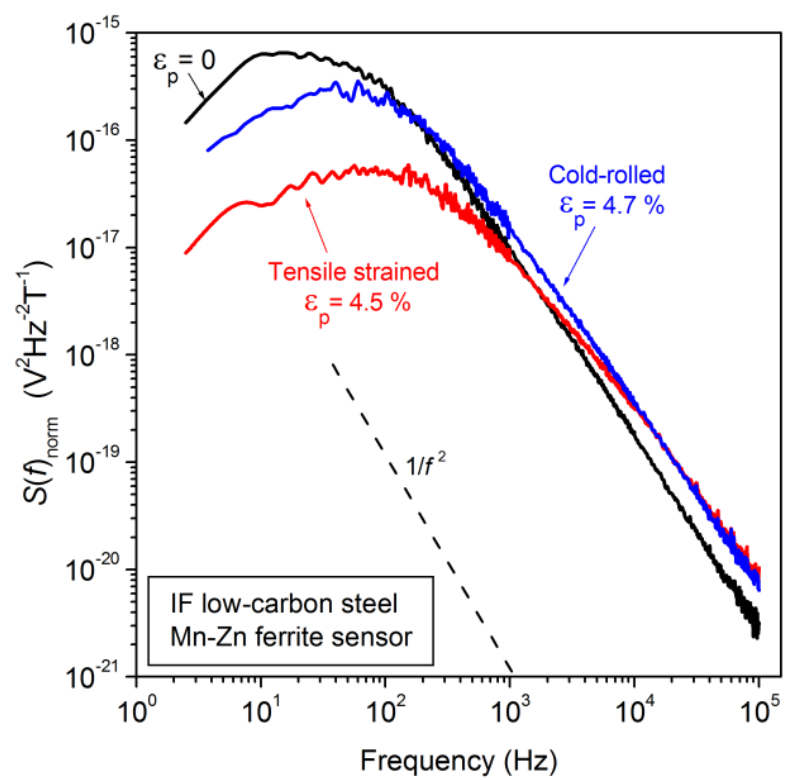

Figure 13. The spectral density of the Barkhausen noise $S(f)$ norm $=S(f) / \mathrm{d} J / \mathrm{d} t$, normalized to the polarization rate, detected by a soft ferrite $\mathrm{C}$-yoke placed on the sheet surface, shows qualitative agreement with the results obtained using search coils, while enhancing the specific differences occurring at low frequencies between cold-rolled and tensile strained samples.

\section{Conclusions}

We have discussed the basic magnetic phenomenology of the plastically deformed steel sheets, with focus on the evolution of hysteresis loop and coercive field with plastic straining and the underlying magnetization process. Plastic deformation by tensile stress or cold-rolling engenders degradation of the soft magnetic behavior of the material, namely the increase of the coercive field and decrease of permeability. This is due to the interaction between the local stress fields of domain walls and dislocations and to the overall modification of the internal energy brought about by the residual stresses and the ensuing rearrangement of the domain structure. It is verified that, for a same permanent elongation, cold-rolling engenders lower detrimental effect on the magnetic properties than tensile straining. The reason apparently lies in the different residual stress pattern associated with the two deformation modes. It is observed, in all cases, that the slope of the hysteresis loops in the second and fourth quadrants behaves in approximately inverse fashion with respect to the increase $\Delta H_{\mathrm{c}}$ of the coercive field due to plastic deformation, so that the loops all cross at a same point in these two quadrants. Such an effect is ascribed to the rise of an equivalent internal demagnetizing factor proportional to $\Delta H_{\mathrm{c}}$. 
Plastic deformation ubiquitously affects the soft magnetic properties of machine cores, because the laminations must be cut before assembling, an operation producing a work-hardened band along the lamination edges. The ensuing increase of the magnetic loss is found to vary with the width $w$ of the cut lamination, according to a $1 / w$ dependence.

The importance of investigating the Barkhausen noise in work-hardened soft magnetic steels is especially recognized in the field of non-destructive testing, where copious literature has appeared and practical testing devices have been proposed. Univocal general conclusions about the effect of plastic straining on BN cannot be drawn, however, because of the variety of exciting conditions and noise analysis methods. We show in this work that methodological and interpretative ambiguities can be removed by supplying the exciting field in such a way that the hysteresis loop is traversed at constant and sufficiently low magnetization rate. The spectral density of the noise $S(f)$ can then be properly analyzed across a wide frequency band. $S(f)$ reveals definite and opposite trends with plastic straining at low and high frequencies. They are related to lifetime and correlation effects of the Barkhausen avalanches and the fine structure of the noise, respectively. Clarifying in this way the noise phenomenology and its relationship with plastic deformation has a certain cost, however, in terms of experimental flexibility and practical application, because very low magnetizing frequencies and defined constant magnetization rates are required. However, there is little alternative to such an approach if unambiguous correlation of the noise with the mechanical effects is pursued.

Acknowledgments: Some of the results by the authors reported in this work were obtained under the contract RFCS-CR-04024 "NUSIMAG", funded by the European Commission.

Author Contributions: This work was the result of a joint activity by the authors, who equally contributed to the study and the writing of the manuscript.

Conflicts of Interest: There are no conflicts of interest of any kind.

\section{References}

1. Bertotti, G.; Fiorillo, F. Crystalline Fe-Si, Fe-Al, and Fe-Si-Al alloys. In Landolt-Börnstein, Magnetic Properties of Metals; Wijn, H.P.J., Ed.; Springer: Berlin/Heidelberg, Germany; New York, NY, USA, 1994; Group III; Volume 19, pp. 33-143, ISBN 3-540-55590-0.

2. Takahashi, N.; Suga, Y.; Kobayashi, H. Recent developments in grain-oriented silicon steel. J. Magn. Magn. Mater. 1996, 160, 98-101. [CrossRef]

3. Gmyrek, Z.; Cavagnino, A.; Ferraris, L. Estimation of the magnetic properties of the damaged area resulting from the punching process: Experimental research and FEM modeling. IEEE Trans. Ind. Appl. 2013, 49, 2069-2077. [CrossRef]

4. Girgis, R.S.; te Nijenhuis, E.G.; Gramm, K.; Wrethag, J.E. Experimental investigations on effect of core production attributes on transformer core loss performance. IEEE Trans. Magn. 1998, 13, 526-531. [CrossRef]

5. Takahashi, S.; Kobayashi, S.; Kikuchi, H.; Kamada, Y. Relationship between mechanical and magnetic properties in cold rolled low carbon steel. J. Appl. Phys. 2006, 100, 113908. [CrossRef]

6. O'Sullivan, D.; Cotterell, M.; Meszaros, I. The characterization of work-hardened austenitic stainless steel by NDT micromagnetic techniques. NDT E Int. 2004, 37, 265-269. [CrossRef]

7. Kleber, X.; Vincent, A. On the role of residual internal stresses and dislocations on Barkhausen noise in plastically deformed steel. NDT E Int. 2004, 37, 439-445. [CrossRef]

8. Liu, T.; Kikuchi, H.; Kamada, Y.; Ara, K.; Kobayashi, S.; Takahashi, S. Comprehensive analysis of Barkhausen noise properties in the cold rolled mild steel. J. Magn. Magn. Mater. 2007, 310, e989-e991. [CrossRef]

9. Manjanna, J.; Kobayashi, S.; Kamada, Y.; Takahashi, S.; Kikuchi, H. Martensitic transformation in SUS 316LN austenitic stainless steel at RT. J. Mater. Sci. 2008, 43, 2659-2665. [CrossRef]

10. Kypris, O.; Nlebedim, I.C.; Jiles, D. Measuring stress variation with depth using Barkhausen signal. J. Magn. Magn. Mater. 2016, 407, 377-395. [CrossRef]

11. Ktena, A.; Hristoforou, E.; Gerhardt, G.J.L.; Missell, F.P.; Landgraf, F.J.G.; Rodrigues, D.L.; Alberteris-Campos, M. Barkhausen noise as a microstructure characterization tool. Physica B 2014, 435, 109-112. [CrossRef] 
12. Park, D.G.; Jeong, H.T.; Hong, J.H. A study on the radiation damage and recovery of neutron irradiated vessel steel using magnetic Barkhausen noise. J. Appl. Phys. 1999, 85, 5726-5728. [CrossRef]

13. Clapham, L.; Jagadish, C.; Atherton, D.L. The influence of pearlite on Barkhausen noise generation in plain carbon steels. Acta Metall. Mater. 1991, 39, 1555-1562. [CrossRef]

14. Palit Sagar, S.; Parida, N.; Das, S.; Dobmann, G.; Bhattacharya, D.K. Magnetic Barkhausen emission to evaluate fatigue damage in a low carbon structural steel. Int. J. Fatigue 2005, 27, 317-322. [CrossRef]

15. Blaow, M.; Evans, J.T.; Shaw, B.A. Surface decarburisation of steel detected by magnetic Barkhausen emission. J. Mater. Sci. 2005, 40, 5517-5520. [CrossRef]

16. Vincent, A.; Pasc, L.; Morin, M.; Kleber, X.; Delnondedieu, M. Magnetic Barkhausen noise from strain-induced martensite during low cycle fatigue of 304L austenitic stainless steel. Acta Mater. 2005, 53, 4579-4591. [CrossRef]

17. Ilker Yelbay, H.; Cam, I.; Hakan Gür, C. Non-destructive determination of residual stress state in steel weldments by Magnetic Barkhausen Noise technique. NDT E Int. 2010, 43, 29-33. [CrossRef]

18. Bilger, H.; Träuble, H. Temperatur und Verformungsabhängigkeit der Koerzitivfeldstärke von Eisen-Einkristallen. Phys. Status Solidi 1965, 10, 755-764. [CrossRef]

19. Traüble, H. The influence of crystal defects on magnetization processes in ferromagnetic single crystals. In Magnetism and Metallurgy; Berkowitz, A.E., Kneller, E., Eds.; Academic Press: New York, NY, USA; London, UK, 1969; Volume 2, pp. 622-687; Library of Congress Catalog Card 69-13488.

20. Küpferling, M.; Appino, C.; Basso, V.; Bertotti, G.; Fiorillo, F.; Meilland, P. Magnetic hysteresis in plastically deformed low-carbon steel laminations. J. Magn. Magn. Mater. 2007, 316, e854-e857. [CrossRef]

21. Swartzendruber, L.J.; Hicho, G.E.; Chopra, H.D.; Leigh, S.D.; Adam, G.; Tsory, E. Effect of plastic strain on magnetic and mechanical properties of ultralow carbon sheet steel. J. Appl. Phys. 1997, 81, 4263-4265. [CrossRef]

22. Astié, B.; Degauque, J.; Porteseil, J.L.; Vergne, R. Influence of the dislocation structures on the magnetic and magnetomechanical properties of high-purity iron. IEEE Trans. Magn. 1981, 17, 2929-2931. [CrossRef]

23. Krause, R.F.; Cullity, B.D. Formation of uniaxial anisotropy in nickel by plastic deformation. J. Appl. Phys. 1968, 39, 356-358. [CrossRef]

24. Perevertov, O. Influence of the residual stress on the magnetization process in mild steel. J. Phys. D Appl. Phys. 2007, 40, 949-954. [CrossRef]

25. Stupakov, O.; Uchimoto, T.; Takagi, T. Magnetic anisotropy of plastically deformed low-carbon steel. J. Phys. D Appl. Phys. 2010, 43, 195003. [CrossRef]

26. Silva, E.M.; Paula, A.M.R.; Leite, J.P.; Leite, J.P.; Andrade, L.S.S.; de Albuquerque, V.H.C.; Tavares, J.M.R.S. Detection of the magnetic easy direction in steels using induced magnetic fields. Metals 2016, 6, 317. [CrossRef]

27. Permiakov, V.; Dupré, L.; Makaveev, D.; Melkebeek, J. Dependence of power losses on tensile stress for Fe-Si nonoriented steel up to destruction. J. Appl. Phys. 2002, 91, 7854-7856. [CrossRef]

28. LoBue, M.; Basso, V.; Fiorillo, F.; Bertotti, G. Power losses and magnetization process in Fe-Si non-oriented electrical steels under compressive and tensile stresses. J. Magn. Magn. Mater. 2000, 215-216, 124-126. [CrossRef]

29. Hattori, N.; Matsumoto, R.; Utsunomiya, H. Residual Stress Distribution through Thickness in Cold-Rolled Aluminum Sheet. Key Eng. Mater. 2014, 622-623, 1000-1007. [CrossRef]

30. Ossart, F.; Hug, E.; Hubert, O.; Buvat, C.; Billardon, R. Effect of punching on electrical steels: Experimental and numerical coupled analysis. IEEE Trans. Magn. 2000, 36, 3137-3140. [CrossRef]

31. Hofman, M.; Naumoski, H.; Herr, U.; Herzog, H. Magnetic properties of electrical steel sheets in respect of cutting: Micromagnetic analysis and macromagnetic modeling. IEEE Trans. Magn. 2016, 52, 2000114. [CrossRef]

32. Crevecoeur, G.; Dupré, L.; Vandenbossche, L.; van de Walle, R. Local Identification of Magnetic Hysteresis Properties Near Cutting Edges of Electrical Steel Sheets. IEEE Trans. Magn. 2008, 44, 1010-1013. [CrossRef]

33. Zhao, H.; Ferrara, E.; Manescu Paltanea, V.; Paltanea, G.; Gavrila, H.; Fiorillo, F. Effect of punching and water-jet cutting methods on magnetization curve and energy losses of non-oriented magnetic steel sheets. Int. J. Appl. Electromagn. Mech. 2017, 55, 69-76. [CrossRef] 
34. Piotrowski, L.; Augustyniak, B.; Chmielewski, M.; Hristoforou, E.V.; Kosmas, K. Evaluation of Barkhausen noise and magnetoacoustic emission signals properties for plastically deformed Armco iron. IEEE Trans. Magn. 2010, 46, 239-242. [CrossRef]

35. Bertotti, G.; Fiorillo, F.; Sassi, M.P. Barkhausen noise and domain structure dynamics in Si.Fe at different points of the magnetization curve. J. Magn. Magn. Mater. 1981, 23, 136-148. [CrossRef]

36. Bertotti, G. Hysteresis on Magnetism; Academic Press: San Diego, CA, USA, 1998; pp. 288-294, ISBN 0-12-093270-9.

37. Küpferling, M.; Fiorillo, F.; Basso, V.; Bertotti, G.; Meilland, P. Barkhausen noise in plastically deformed low-carbon steels. J. Magn. Magn. Mater. 2008, 320, e527-e530. [CrossRef]

38. Bertotti, G.; Fiorillo, F.; Rietto, A.M. Microscopic and macroscopic aspects of the magnetization process in Si-Fe single crystals. IEEE Trans. Magn. 1984, 20, 1481-1483. [CrossRef]

(C) 2017 by the authors. Licensee MDPI, Basel, Switzerland. This article is an open access article distributed under the terms and conditions of the Creative Commons Attribution (CC BY) license (http:// creativecommons.org/licenses/by/4.0/). 